\title{
Transiciones a la educación posobligatoria en Madrid: el peso de los factores políticos e institucionales
}

\section{Miriam Prieto Egido}

Universidad Autónoma de Madrid. Departamento de Pedagogía

miriam.prieto@uam.es

Javier Rujas Martínez-Novillo

Universidad de Burgos. Área de Sociología

jrujas@ubu.es

Recepción: 18-10-2019

Aceptación: 27-01-2019

Publicación: 01-04-2020

\section{Resumen}

El presente artículo analiza los condicionantes del acceso a la educación posobligatoria (Bachillerato y Formación Profesional de Grado Medio) en Madrid. Para ello, se basa en el análisis de entrevistas a agentes políticos, educativos y empresariales, de la normativa y de datos de fuentes secundarias. Sitúa, primero, la educación posobligatoria en Madrid en su contexto local y en el marco de las políticas educativas desarrolladas en la región. A continuación, analiza el peso específico de los factores políticos e institucionales en las transiciones a esta etapa desde la ESO. Muestra cómo el diseño de la oferta y la admisión, sujetos a distintos condicionantes, difieren en ambas vías, ya que en FP se produce una tensión entre las «necesidades del mercado" y la demanda social que no se da en Bachillerato. Por último, analiza los condicionantes de la elección de estudios posobligatorios. Concluye que las dos ramas de la posobligatoria no pueden entenderse de forma independiente: la vía profesional suscita más atención pública y debate que la académica, mayoritaria y apenas cuestionada; la diferencia de prestigio se mantiene, y afecta de forma distinta a los diferentes niveles de FP y a sus familias y ciclos. La configuración de la oferta y el diseño institucional, en interacción con otros factores (sociales, subjetivos, territoriales, culturales), condicionan las elecciones y transiciones educativas y contribuyen a generar desigualdades educativas.

Palabras clave: educación posobligatoria; política educativa; transiciones educativas; oferta educativa; Bachillerato; Formación Profesional; transiciones educativas 
Abstract. Transitions to post-compulsory education in Madrid: The weight of political and institutional factors

This paper examines the factors that influence access and choice in post-compulsory education in Madrid (baccalaureate and intermediate vocational training), drawing on an analysis of qualitative interviews with political, educational and corporate agents, as well as education regulations and secondary data. First, the paper describes post-compulsory education in Madrid focusing on the local context and educational policies implemented in the region. Second, the specific weight of political and institutional factors in post-compulsory transitions is addressed. The paper shows how the design of educational provision and admissions in both branches is differentiated and influenced by different factors: vocational training is affected by the tension, absent in the academic track, between market needs and young people's interests. Finally, the paper analyses the factors influencing post-compulsory education choices. The paper concludes that both tracks cannot be understood separately: vocational training is far more present in the public debate than the baccalaureate, which remains hegemonic and almost unquestioned. Differences in the prestige of both tracks also persist, which affect the various levels, families and degrees of vocational training differently. The configuration of educational supply and the institutional design of both tracks, in interaction with other factors (social, subjective, territorial, cultural), conditions choices and transitions, leading to educational inequalities.

Keywords: post-compulsory education; education policy; educational provision; baccalaureate; vocational training; educational transitions

\section{Sumario}

1. Introducción $\quad 5$. Los condicionantes del acceso a la

2. Transiciones educativas a la educación educación posobligatoria en la ciudad posobligatoria: factores políticos e institucionales

\section{Metodología}

4. La educación posobligatoria en Madrid: una diferenciación institucional, material y simbólica de Madrid

6. La elección de estudios posobligatorios: ¿una decisión libre?

\section{Conclusiones}

Financiación

Referencias bibliográficas

\section{Introducción}

El final de la educación obligatoria marca un momento clave en las trayectorias escolares y sociales de los jóvenes. En este punto, se dibujan diversos caminos con distinto valor social que conducen a distintas experiencias y oportunidades vitales. Aunque la expansión educativa en España llevó a la progresiva generalización de la educación secundaria superior, este aumento se produjo sobre todo en el Bachillerato - aunque la Formación Profesional (FP) también ha crecido en las últimas décadas, especialmente durante la crisis- y no supuso, sin embargo, su universalización: mientras la educación obligatoria la cursa prácticamente todo el alumnado, a los 16 años la tasa de escolarización des- 
ciende al 95,6 \%; a los 17 años al 88,7 \%, y a los 18 años al 75,3 \% (MEFP, 2019a). Esto, junto con la inclusión en la agenda europea de la reducción del abandono escolar temprano como objetivo prioritario de la Estrategia Europa 2020, ha puesto el foco de atención en el momento de paso la Educación Secundaria Obligatoria (ESO) al Bachillerato y a la FP.

Las transiciones educativas han ido adquiriendo progresivamente más atención en la investigación social y educativa, ya que constituyen un momento decisivo en la construcción y en la reproducción de desigualdades (Tarabini e Ingram, 2018). Además, las transformaciones sociales de las últimas décadas, como la extensión de los periodos de escolarización, la diversificación de los estilos de vida o la flexibilización del mercado laboral, han generado cambios en las trayectorias educativas y las han hecho más diversas y plurales (Walther, 2006). Esta "desestandarización», sin embargo, no implica que no existan regímenes constituidos por patrones institucionales y valores culturales que configuran lo que se entiende por normal en un contexto determinado y condicionan las decisiones del alumnado. Es, por tanto, imprescindible analizar cómo se construyen las transiciones educativas de los jóvenes a la educación secundaria posobligatoria y los factores (socioeconómicos, culturales, territoriales, institucionales, subjetivos) que pesan sobre ellas.

En este proceso tienen un valor específico factores políticos e institucionales que pueden adoptar distintas configuraciones según los contextos locales y las políticas educativas implementadas. Este artículo analiza los condicionantes del acceso a la educación posobligatoria, con una especial atención al diseño y la planificación de la oferta de Bachillerato y Formación Profesional de Grado Medio (FPGM), y los condicionantes de las elecciones escolares en la transición a esta etapa en Madrid. Para ello, se basa en entrevistas a agentes políticos, educativos y empresariales, en el análisis de la normativa que regula la educación posobligatoria en la Comunidad de Madrid (CM) y en datos cuantitativos de fuentes secundarias.

\section{Transiciones educativas a la educación posobligatoria: factores políticos e institucionales}

En la etapa que sigue a la finalización de la educación obligatoria, la mayoría de los sistemas educativos mantienen la distinción entre una rama académica, que prepara a los estudiantes para el acceso a la universidad, y una profesional, que prepara para la entrada al mercado laboral. Numerosos estudios han mostrado que esta diferenciación institucional está asociada a un sesgo de clase, pues el origen social familiar pesa en el camino escogido al acabar la educación obligatoria (Ball et al., 2000; Brunila et al., 2011; Lahelma, 2009; Shavit y Müller, 2000). El caso español no es una excepción: el origen social influye tanto en el riesgo de fracaso escolar (Calero et al., 2010) como en la elección entre la rama académica y la profesional de la educación posobligatoria (Calero, 2008; Bernardi y Requena, 2010; Elias y Daza, 2017). Los jóvenes de clases medias y altas tienen mayores probabilidades de cursar Bachillerato que los de 
clases trabajadoras, que tienen mayor probabilidad de abandonar el sistema educativo o de elegir la enseñanza profesional, que requiere menor inversión económica y de tiempo. Esta les ofrece mejores oportunidades laborales a corto plazo, para evitar así el desempleo y las ocupaciones menos cualificadas, aunque al precio de reducir sus posibilidades de acceder a la universidad (Bernardi y Requena, 2010).

Por ello, las transiciones educativas desde la etapa obligatoria a la posobligatoria constituyen no solo un momento vital clave, sino también un aspecto central a la hora de promover la equidad en los sistemas educativos y la igualdad de oportunidades de todo el alumnado. Estas son el resultado de un complejo entramado de factores, entre los que se encuentran intereses, expectativas, proyecciones del alumnado y sus familias, así como factores socioeconómicos, étnicos, de género, institucionales y culturales (Walther, 2006). En un contexto histórico y cultural en el que la globalización y las ideologías neoliberales refuerzan la concepción de las elecciones como una cuestión individual, basada únicamente en las capacidades y las preferencias del individuo, en la que se niegan y ocultan las diferencias estructurales (Ball, 2006; Brunila et al., 2011), el análisis de los condicionantes institucionales es clave a la hora de comprender las transiciones educativas del alumnado.

Si bien todas las transiciones educativas son cruciales, ya que constituyen momentos de cambio y posible incertidumbre, la transición a la educación posobligatoria tiene un riesgo y un valor añadido en el sistema educativo español, por ser la primera en la que el alumnado puede no continuar los estudios. Además, el aumento de los niveles de cualificación requeridos en el mercado de trabajo hace necesario ir más allá de la educación obligatoria para garantizar mejores oportunidades laborales. Especial atención ha recibido, además, esta transición en la prevención del abandono escolar temprano impulsada por la estrategia Europa 2020. En esta línea, la última reforma del sistema educativo español introdujo cambios sustanciales que influyen de forma determinante en el paso de la educación obligatoria a la posobligatoria y que conducen a la construcción de una "cultura de la diferenciación" (Dupriez y Dumay, 2006). La denominada flexibilización de las trayectorias, con la introducción de dos itinerarios diferenciados en el último curso de la ESO y la creación de los Programas de Mejora del Aprendizaje y el Rendimiento (PMAR) o la FP Básica, puede ser determinante en la segregación del alumnado entre las ramas académica y profesional (Tarabini y Montes, 2015). Estas medidas vienen a sumarse a un contexto que ha sido calificado como un "régimen no protector» (Walther, 2006), caracterizado por altas tasas de abandono escolar temprano, pobre desarrollo de las enseñanzas profesionales y sobrecualificación, en el que los sistemas de transición ofrecen pocas opciones, flexibilidad y/o seguridad, y dependen en gran medida del apoyo familiar.

A estos factores sistémicos (regímenes de protección social y estructura del sistema educativo) se unen factores políticos (diseño y desarrollo de la oferta, organización del proceso de admisión, creación de programas y modalidades especiales, etcétera) e institucionales, como la organización de los propios cen- 
tros, los modos de gestión de la heterogeneidad del alumnado, las prácticas de orientación y las formas de canalización y enmarcado de las trayectorias y elecciones de los jóvenes (Tarabini e Ingram, 2018; Tarabini et al., 2018). Algunos de estos factores políticos e institucionales, en particular los cambios en la arquitectura del sistema y sus efectos, se han analizado en clave histórica (Merino, 2013), y estudios recientes destacan que los factores institucionales, como la titularidad de centro, a su vez asociada a una determinada composición social, a distintas dinámicas institucionales y mecanismos de evaluación, pesan en la elección de itinerario posobligatorio: en los centros privados-concertados la presión por estudiar Bachillerato sería mayor (Elias y Daza, 2017). No obstante, escasean análisis detallados de las políticas educativas posobligatorias que ahonden en las tensiones y los condicionantes que las atraviesan y en su configuración a nivel regional y local.

Las políticas educativas nacionales, traducidas y adaptadas a nivel local, contribuyen a enmarcar y encauzar las elecciones y decisiones educativas de los jóvenes en función de ciertas ideas y concepciones políticas de las distintas enseñanzas, instrumentos de canalización del alumnado y arreglos institucionales establecidos con docentes y escuelas (van Zanten et al., 2018). A su vez, estas políticas constituyen espacios de conflicto entre actores con posiciones e intereses diversos: son inestables, cambiantes, están atravesadas por distintas lógicas y tensiones, y tienen efectos ambivalentes, a veces perversos y diferentes de los previstos (Ball et al., 2012; Martín Criado, 2010). Se definen tanto por lo que se hace como por lo que no se hace - no hacer política es una forma de hacer política-, tanto por lo que se prioriza como por lo que se omite o se excluye de la agenda (Bonal, 2012).

Además, su realización en la vida cotidiana de las escuelas implica traducciones, recontextualizaciones y reajustes mediados por factores institucionales (condiciones sociales y materiales de los centros, ideologías pedagógicas, ethos y cultura de la escuela o habitus institucional) (Ball et al., 2012). En este sentido, el desarrollo y la configuración de la oferta educativa y los procesos de orientación y construcción de la «demanda» de educación están interrelacionados de forma compleja. Los efectos de las políticas nacionales y regionales se combinan, refuerzan o contradicen, así, con el efecto específico de los centros educativos, sus prácticas y arreglos institucionales, y los propios deseos y aspiraciones de los jóvenes y sus familias, en los procesos de elección de estudios posobligatorios.

\section{Metodología}

Este trabajo se basa en una serie de entrevistas a actores clave en el diseño y la oferta de educación posobligatoria en la ciudad de Madrid, complementado con un análisis del marco normativo y de datos cuantitativos secundarios. La combinación de estos tres elementos ha permitido recoger información cuantitativa y cualitativa sobre la situación de la educación posobligatoria en Madrid: su organización, funcionamiento y características en el ámbito regional 
y el local (resultados presentados en el apartado 4). Más específicamente, las entrevistas han permitido también (1) identificar las lógicas, las prácticas y los criterios manejados en el diseño de la oferta posobligatoria, (2) sus tensiones y condicionantes y sus efectos en las elecciones educativas, a través de los discursos de los distintos agentes implicados en la política educativa en esta etapa (resultados expuestos en los apartados 5 y 6 ).

Las entrevistas tienen, por tanto, un doble objetivo, a la vez informativo y de análisis de los discursos expertos sobre esta etapa. Ofrecen información de primera mano sobre el proceso de planificación y organización de la oferta educativa y los criterios y razones de los policymakers, con frecuencia no reflejados en los textos oficiales - especialmente en un contexto de falta de transparencia y ausencia de datos públicos como el que analizamos. Sirven, así, para recomponer las lógicas que hay detrás de las políticas educativas en Bachillerato y FPGM (elaboración y planificación) y sus dificultades reales (implementación), pero también para entender cómo se representan los actores clave la situación de estas dos opciones escolares y la transición a ellas desde la ESO (sus retos, dificultades y soluciones en materia de planificación, organización de la oferta, distribución territorial, relación con las demandas sociales y empresariales, etcétera).

Se han realizado 13 entrevistas en profundidad a agentes políticos, educativos y empresariales seleccionados por su conocimiento de la situación de la educación posobligatoria en Madrid (ver cuadro 1). La inclusión en la muestra de agentes relevantes externos a la Administración, además de necesaria por la dificultad de acceso a esta, es fundamental para entender las pugnas en materia de educación posobligatoria e identificar los consensos, conflictos, tensiones y omisiones que se dan en este ámbito. Varios entrevistados, además de ser miembros de la Administración, de organizaciones educativas o sindicales, cuentan con largas trayectorias en docencia, cargos directivos o inspección, lo

Cuadro 1. Muestra de entrevistas

\begin{tabular}{lll}
\hline & Entrevistados & Código verbatim \\
\hline Agentes políticos y administrativos * & Cargos Administración educativa (2) & CA, CA-FP \\
& Técnicos Administración educativa (2) & TA \\
& Sindicatos (3) & SE, SFP \\
\hline Agentes educativos & Asociación de directores (2) & AD, AD-FP \\
& Asociación de familias (1) & AF \\
& Asociación de orientadores (1) & AO \\
& Organización de escuelas privadas (1) & AEP \\
\hline Agentes empresariales & Patronal local (1) & PL \\
\hline
\end{tabular}

* Debido a la complejidad y la dificultad que supone contactar con algunos agentes de la Administración, por su carga de trabajo y sus reticencias a ser investigados, a pesar de reiterados intentos, se ha logrado entrevistar a un cargo de la Administración educativa del ámbito de la FP, pero no se ha conseguido en el caso del Bachillerato y la organización de la enseñanza concertada y privada (dependientes de otras direcciones generales de la Consejería de Educación).

Fuente: elaboración propia 
que permite contrastar la visión oficial con visiones más cercanas a los contextos materiales y sociales concretos de los centros educativos. Partiendo de categorías básicas que se fueron matizando y completando inductivamente, el análisis cualitativo permitió comparar las distintas percepciones y los posicionamientos, identificar las prácticas y lógicas subyacentes a la política educativa y analizar los discursos con relación a la posición de los hablantes en el campo escolar y con el contexto social y educativo.

Aunque nuestro estudio se centra en Madrid capital, la concentración de las competencias educativas en el nivel autonómico hace necesario referirse también al conjunto de la región. Además, Madrid capital concentra el $45 \%$ de la educación secundaria posobligatoria de la CM y muchos datos son muy similares a nivel regional y municipal, por lo que los resultados estadísticos que presentamos se refieren globalmente a la CM y precisamos cuando la capital presenta características específicas.

\section{La educación posobligatoria en Madrid: una diferenciación institucional, material y simbólica}

A pesar de registrar una tasa de abandono escolar temprano (14,4\%) por debajo de la media nacional (17,9\%) (MEFP, 2019b: 28), la Comunidad de Madrid es la región española con menor gasto público por alumno y mayor gasto de las familias en educación (Comunidad de Madrid, 2019: 102 y 114), con mayor número de alumnos por profesor (MEFP, 2019b: 15), la segunda con mayor porcentaje de alumnado de enseñanzas no universitarias escolarizado en el sector privado (84,2\%) (MEFP, 2019b: 15) y la primera en segregación escolar (Murillo y Martínez-Garrido, 2018). Entre 2003 y 2015, la región llevó a cabo un proceso de reforma global del sistema educativo caracterizado por la introducción de medidas de rendición de cuentas, libertad de elección y autonomía de los centros escolares. Estas políticas, sin embargo, acentuaron la competencia entre centros, limitaron su autonomía y promovieron un imaginario en el que la escolarización es resultado de las elecciones «libres» del alumnado o sus familias que choca con la realidad de una elección fuertemente condicionada por el diseño institucional del sistema educativo (Prieto y Villamor, 2012; 2018).

En el caso de la educación posobligatoria, la CM se caracteriza por una alta proporción de titulados en educación secundaria de segunda etapa, aunque con un gran desequilibrio entre la rama académica y la profesional. Aunque el $75 \%$ de la población de 20 a 29 años ha completado al menos este nivel (Ruiz et al., 2019: 40), la CM es una de las regiones españolas con mayor proporción de personas con estudios superiores $(47,2 \%)^{1}$ y con la tasa bruta de escolarización en CFGM más baja de España (26,7 \%, frente a la media nacional, de 35,6 \%) (Ruiz et al., 2019: 36). Las enseñanzas posobligatorias han crecido, como en el resto de España, desde los primeros años de la década del 2000,

1. Fuente: MEFP, Explotación de las variables educativas de la Encuesta de Población Activa. Nivel de formación de la población de 25-64 años. 
Gráfico 1. Evolución del alumnado de posobligatoria por rama en España y la CM (20022017). Porcentajes

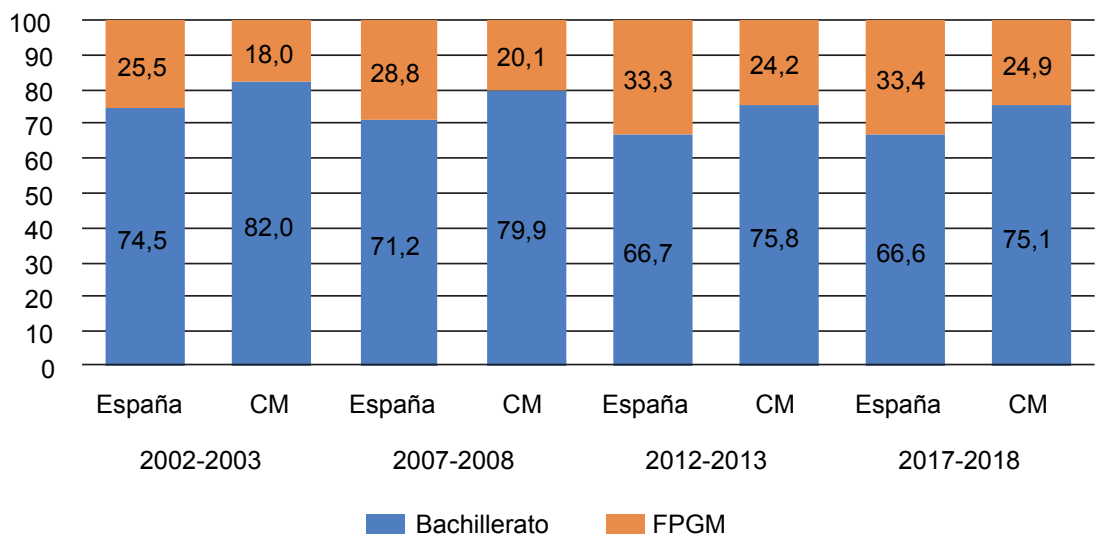

Fuente: elaboración a partir de MEFP, Estadística de las enseñanzas no universitarias.

especialmente durante la crisis económica y más en FP que en Bachillerato. No obstante, aunque el desequilibrio entre ambas vías se ha reducido ligeramente, se mantiene con fuerza (gráfico 1), en una proporción de tres estudiantes y centros de Bachillerato por cada alumno y centro de FPGM (calculado a partir de Comunidad de Madrid, 2019). Además de esta opción muy mayoritaria por el Bachillerato, en Madrid se da un fuerte peso de la red privada, intensificado en los últimos años. La red pública acoge a la mayoría del alumnado de Bachillerato y FPGM, pero en una proporción inferior a la media nacional; en número de centros, la red privada es mayoritaria en ambas enseñanzas, aunque en la rama académica predomina la privada y en la profesional la concertada, que en la capital ha superado en los últimos cursos a la pública (tabla 1).

En esta etapa, la Comunidad de Madrid también ha desarrollado programas que han generado diferenciaciones internas en cada rama de la poso-

Tabla 1. Centros y alumnado de Bachillerato y FPGM por titularidad en España, CM y Madrid capital (curso 2016-2017). Porcentajes

\begin{tabular}{|c|c|c|c|c|c|c|c|}
\hline & & \multicolumn{2}{|c|}{ España } & \multicolumn{2}{|c|}{ C. de Madrid } & \multicolumn{2}{|c|}{ Madrid capital } \\
\hline & & Bachillerato & FPGM & Bachillerato & FPGM & Bachillerato & FPGM \\
\hline \multirow[t]{3}{*}{ Centros } & Público & 67,1 & 71,2 & 43,8 & 80,3 & 30,3 & 38,7 \\
\hline & Privado-concertado & 10,1 & 19,6 & 6,5 & 35,4 & 11,9 & 43,4 \\
\hline & Privado & 22,8 & 9,2 & 49,8 & 12,6 & 57,8 & 17,0 \\
\hline \multirow[t]{3}{*}{ Alumnado } & Público & 74,0 & 73,6 & 62,2 & 63,6 & 48,0 & 55,7 \\
\hline & Privado-concertado & 10,9 & 22,1 & 7,4 & 29,7 & 13,8 & 35,6 \\
\hline & Privado & 15,1 & 4,4 & 30,4 & 6,8 & 38,2 & 8,7 \\
\hline
\end{tabular}

Fuente: elaboración a partir de MEFP, Estadística de las enseñanzas no universitarias (España), Comunidad de Madrid (2018) y Banco de Datos del Ayuntamiento de Madrid. 
bligatoria, todos ellos minoritarios, pero en expansión. En 2012, se creó el Bachillerato de Excelencia, un programa con alto nivel de exigencia dirigido al alumnado con buen expediente en la ESO. Se desarrolla en la actualidad en un centro de excelencia y 14 aulas de excelencia en IES, con un número reducido de alumnos (867, previsión 2018-2019) (Comunidad de Madrid, 2018: 39). El programa bilingüe español-inglés, por su parte, se desarrolla fundamentalmente en Primaria y ESO, pero se ha implementado también en Bachillerato, pasando en los últimos cinco cursos de 32 a 98 centros y de 3.586 a 19.578 alumnos. En FP, empezó en 2016-2017, pero solo afecta a 9 centros y 311 alumnos, y a seis ciclos de Grado Superior (Comunidad de Madrid, 2018). Existen, además, otras formas de diferenciación, como las secciones lingüísticas de francés y alemán en Bachillerato, en una minoría de institutos (11 y 4 respectivamente) (Comunidad de Madrid, 2018: 35), o el Bachillerato Internacional y el Bachillerato Dual Americano, en crecimiento en los últimos años. En FP, Madrid ha sido de las regiones que más han apostado por la FP Dual (Ruiz et al., 2019: 38), aunque sigue siendo una modalidad minoritaria desarrollada fundamentalmente en Grado Superior. Todo ello refleja y refuerza la priorización de la rama académica de la posobligatoria, en general, y, en el caso de la FP, de su nivel superior, más prestigioso y dotado de recursos que el Grado Medio.

Ambas ramas difieren también en el proceso y los criterios de admisión. En 2013, se implantó el llamado «distrito único» con el fin de promover la «libertad de elección»: todo el territorio de la CM pasó a ser considerado como zona única de escolarización (Decreto 29/2013). Este cambio afectó a Bachillerato, pero no a la FP, que, en la práctica, por la escasez de oferta y la dispersión de las especialidades, ya funcionaba como zona única. En Bachillerato, cuando la demanda de plazas supera a la oferta en un centro, se prioriza en función de la presencia de hermanos en este, la cercanía del domicilio familiar o el lugar de trabajo de los padres, la renta anual familiar, la discapacidad en los padres o hermanos, y la situación de familia numerosa, a los que se suma la nota media del expediente académico del último curso de ESO (Decreto 11/2019). En el caso de la FPGM, por el contrario, se tienen en cuenta únicamente criterios académicos (nota media y reconocimientos académicos en ESO) (Orden 2509/2013), no económicos o familiares.

Antes de 2015, la planificación y la organización de las enseñanzas de Bachillerato y FP estaban unificadas en una misma dirección general (DG) de la Consejería de Educación. A partir de ese año, no obstante, se separaron: se dotó a la FP de una DG propia (DG de FP y Enseñanzas de Régimen Especial); y el Bachillerato pasó a depender de la DG de Educación Infantil, Primaria y Secundaria (Decreto 72/2015). ${ }^{2}$ Asimismo, en 2016, tras una déca-

2. Esta organización rige para los centros públicos. Los centros privados y los conciertos educativos dependen a su vez de otra DG. Tras las elecciones autonómicas de 2019, el nuevo gobierno de la CM ha reformado su estructura orgánica, y ha vuelto a unificar la planificación y la organización de secundaria y FP en una misma DG. 
da de inactividad, se reactivó el Consejo de FP, que reúne a actores políticos, sindicales y empresariales para orientar el diseño de esta enseñanza. Esta diferenciación organizativa, que se justifica por las particularidades de la FP y por una voluntad de revalorizarla y dotarla de mayor entidad propia, se traduce en una planificación separada y refleja a su vez la "continuidad material y simbólica entre Bachillerato y ESO» (Tarabini y Jacovkis, 2019: 253).

\section{Los condicionantes del acceso a la educación posobligatoria en la ciudad de Madrid}

Las políticas educativas en materia de libertad de elección desarrolladas en la $\mathrm{CM}$ han contribuido a crear un imaginario que reduce el acceso a la educación a una cuestión individual y subjetiva, pasando por alto el papel de la configuración de la oferta y el diseño institucional de las enseñanzas. Sin embargo, nuestros resultados muestran que existe una estrecha y compleja relación entre la oferta y la demanda de educación posobligatoria en Madrid, que está fuertemente condicionada por factores institucionales y adopta distintas formas en ambas ramas.

\subsection{El diseño de la oferta en Bachillerato: ¿guiado por la demanda?}

Según el sistema de libertad de elección existente en Madrid, la oferta de plazas en Bachillerato, especialmente cuando se trata de la ampliación de unidades en los centros públicos o impartición de asignaturas optativas, queda sujeta a la demanda. Así, la Administración fija los grupos que tendrá cada centro antes de iniciar el proceso de escolarización, y supedita la apertura de nuevos grupos a la existencia de solicitudes. Sin embargo, estas tienen que alcanzar un número mínimo. Por otro lado, la autorización de nuevos grupos y la asignación de los alumnos que quedan sin plaza en el proceso ordinario de escolarización (junio) se hacen en el proceso de escolarización extraordinario de septiembre. Ambos aspectos dejarían, según algunos entrevistados, a muchas familias en una situación de incertidumbre que favorecería el trasvase de alumnado al sector privado, lo que dejaría a los centros públicos en desventaja.

En 2017-2018, 2.362 alumnos no obtuvieron plaza en Bachillerato en ninguno de los seis centros - públicos o concertados- incluidos en su solicitud inicial en la fase de asignación provisional (si la cifra se refiriese al centro escogido en primera opción, sería mucho más alta) (CCOO Madrid, 2019: 18). Estos estudiantes sin plaza, concentrados mayoritariamente en primer curso y en las zonas con más población escolar - Madrid capital (778) y la zona sur $(763)^{3}$ (ibid.) —, pueden obtenerla más adelante tras reclamar o solicitar plaza en otros centros, pero no se ofrecen listas de vacantes y deben esperar hasta septiembre para poder solicitar plaza en otro lugar, además deben ir centro por

3. La Consejería de Educación de la CM divide la región en cinco direcciones de área territorial (DAT): Madrid Capital, Madrid Este, Madrid Oeste, Madrid Norte y Madrid Sur. 
centro. Si tampoco así obtienen plaza, se les busca una en el proceso extraordinario de escolarización y con frecuencia se recurre a subidas de ratio en los grupos. La insuficiencia de plazas y la complejidad del proceso generan, así, una movilidad obligada en el alumnado.

Ha habido últimamente, con el tema de la crisis, una derivación de los alumnos hacia la enseñanza pública, porque en Madrid sabes que el Bachillerato está concertado en muy poquitos centros, en la mayoría de los centros es privado, entonces hay muchas familias que tienen a los alumnos hasta secundaria en un centro concertado y después solicitan plazas en un centro público [...] Hemos notado un aumento de solicitudes y los centros públicos estamos muy saturados [...] No éramos capaces de dar respuesta a esta solicitud de los alumnos $\mathrm{y}$ al final muchos padres terminan haciendo un sacrificio y los llevan a un concertado que saben seguro en junio si tienen plaza. (AD)

Así, en los últimos años, se ha producido un doble movimiento que la ausencia de datos oficiales no permite cuantificar. Por un lado, parte de los estudiantes escolarizados en centros concertados pasan a centros públicos en la transición al Bachillerato coincidiendo con el fin de los conciertos. Por otro lado, hay un trasvase de alumnado al sector privado debido a la insuficiencia de la oferta pública en Bachillerato y su saturación durante el periodo de crisis, en el que aumentó la demanda de la escolarización.

El hecho de que la Administración educativa condicione la oferta a la demanda hace, además, que la diversidad de la oferta que postula la actual ley educativa no sea real, ya que no se hace efectiva cuando las enseñanzas (modalidades de Bachillerato u optativas) no llegan a alcanzar la demanda mínima fijada por la Consejería.

La opcionalidad que al menos en teoría debería de existir, no existe. Claro, esto provoca que haya un baile, un movimiento de alumnos inmenso. Porque, primero, por ejemplo, Bachillerato de Artes tenemos muy pocos en Madrid y prácticamente todos ubicados en Madrid capital [...] En las materias optativas, la cosa se complica todavía más, porque en teoría pueden ser muy amplias, en la práctica son escasísimas. Claro, esto lo que provoca es una movilidad dentro del alumnado de la pública impresionante, porque tú puedes estar en un centro donde no tienes la opción o la modalidad de Bachillerato que quieres, con lo cual o te quedas estudiando un itinerario que no es el tuyo o tienes que buscarte otro centro. (SE)

O sea tú les abres el mundo a las posibilidades, pero luego dices: «Ahora te lo cierro porque, mira, no me sale el grupo». Claro, luego, de repente, en septiembre te llegan alumnos: «Es que mira, es que estoy matriculada en tal centro, pero me han dicho que no sale el grupo de Dibujo Técnico». Entonces, es el alumno quien tiene que andar buscando por ahí si hay algún centro donde ofertan Dibujo Técnico. Hay veces que lo suplimos entre los directores [...] pero es una faena para el alumno, un alumno que quiere hacer una determinada modalidad de enseñanza, luego que quiere acceder a un grado 
[...] que no pueda cursar el Bachillerato porque no hay suficiente número de alumnos. (AD)

Esta optatividad «ficticia», limitada por la demanda, condicionaría las decisiones educativas de los estudiantes y su desarrollo académico, y generaría movilidad o expectativas frustradas en el alumnado por no poder cursar las opciones que desean. Además, en esta lógica de planificación se refleja una desigualdad entre centros más grandes y demandados, que pueden permitirse una mayor variedad de oferta, y otros centros de menor tamaño y demanda, con dificultades para mantener ciertas optativas.

No tengo capacidad para matricular a todo el alumnado que me solicita el centro [...] porque yo matriculo a los alumnos que provienen de mi secundaria, pero luego tengo como un 50 o $60 \%$ de alumnos que me vienen de fuera, de los centros concertados, o incluso de centros públicos que no pueden ofertar toda la variedad que hay en el Bachillerato. Claro, al tener cinco grupos, te es mucho más fácil tener una oferta amplia y, por ejemplo, Dibujo Técnico está en muy poquitos centros, o Tecnología, yo puedo mantenerla todavía porque tengo mucho alumnado. (AD)

Cabe preguntarse, por tanto, si esta lógica de planificación de la oferta centro a centro, según demanda mínima, puede contribuir a reproducir y profundizar esta desigualdad entre centros y generar una especie de efecto Mateo (más demanda y oferta en los centros más demandados y con más oferta, y menos para los que menos tienen de partida).

\subsection{El diseño de la oferta de FP: ¿una planificación sin plan?}

La oferta de FP entraña una mayor complejidad con relación a la de Bachillerato, por motivos idiosincrásicos de estas enseñanzas: la diversidad de familias existente, su mayor coste económico y su relación con el mercado laboral. Sobre el primer aspecto, existen 23 familias de FP en Madrid, lo que dificulta su presencia en todos los distritos y barrios, a diferencia de las tres modalidades de Bachillerato. Además, las distintas familias y ciclos difieren no solo en el tipo de estudios y la profesión a la que dan acceso, sino también en el coste de las enseñanzas: unas requieren pocas infraestructuras (por ejemplo, ciclos de carácter administrativo), y otras requieren instalaciones, equipamientos y recursos específicos y costosos.

Toda la rama de Imagen y Sonido está muy solicitada y hay poca oferta, las de sanitarios también [...] Claro, se necesita estar asociado a un centro hospitalario para poder hacer esas prácticas, entonces ahí la oferta sí que es menor, pero, vamos, en principio te pasa lo mismo en Bachillerato. No es que haya más oferta, es que el Bachillerato es el Bachillerato, hay muchos centros y se oferta el mismo Bachillerato en todos los sitios; en FP está mucho más diversificado. Hay especialidades que quedan vacantes, no muchas, pero hay especialidades que están saturadas. (AD) 
Más allá de la variedad de familias y ciclos, que hace complejo el diseño de la oferta, en las entrevistas aparece de forma clara el peso de la planificación - o su ausencia - en el diseño de la oferta de FP en Madrid, aunque con disensos significativos en su valoración y sobre los criterios en que se basa. Los sectores más críticos afirman que las decisiones se toman a corto plazo, sin un análisis previo de la realidad (de los intereses del alumnado y las demandas del mercado laboral), sin consideración de las desigualdades territoriales y con un ritmo lento de la Administración a la hora de responder a las innovaciones y nuevas demandas.

Pero, vamos, no tienen ningún plan. $\mathrm{O}$ sea, se ha ido haciendo un poco por inercia. O sea, el instituto tal tenía, pues iba ofreciendo. [...] como tengo Grado Superior hago Medio, como tenía Grado Medio, ahora oferto Grado Superior. Entonces resulta que puede ser... [...] por propias instalaciones y demás. Es decir que no hay un plan que digas, yo qué sé, «aquí en el corredor del Este y en la zona Sur, que son gente de menos nivel, vamos a hacer más Grado Medio porque nos van a llegar menos al Grado Superior, porque las tasas están en los superiores, y además lo vamos a hacer en segmentos productivos que están implantados en esa zona», ¿̨no? (S-FP)

En ausencia de dicha planificación, las decisiones se tomarían, según los críticos, siguiendo un criterio de ahorro económico, lo que tiende a generar concentración de la oferta de FP, ya que los recursos existentes (instalaciones, maquinaria, etcétera) se emplean como criterio para la ubicación de las nuevas titulaciones que se aprueban.

Es una negociación centro-Administración y basada básicamente en coste de oportunidad [...] Ya das Grado Superior y te dicen «te doy un concierto de Grado Medio, te puedo mantener el de Grado Superior pero tienes que hacer este Grado Medio», o "te mantengo el de Grado Medio pero hazme esta FP Básica». Entonces, si tú tienes cosas de Electricidad y te digo "hazme una FP Básica de Cocina», dices: «Joder, tengo que montar la cocina...». Para amortizar eso es muy complicado. Entonces, al final acaban reproduciendo la oferta de lo que ya tienes. (S-FP)

Esta lógica de planificación ad hoc, centro a centro y en función de los recursos previos, sin un plan a largo plazo y sin un análisis detallado de sus condicionantes y consecuencias, refuerza la inercia institucional y tiende reproducir el mapa de la oferta existente, con sus desigualdades territoriales y entre centros. Constituye, así, un ejemplo de politics of non-decision-making (Bonal, 2012) que no encara las desigualdades existentes entre las ramas posobligatorias (y dentro de cada una) y las inequidades en el acceso que generan. Para romper con esas inequidades, los agentes educativos reclaman la introducción de un criterio territorial en la distribución de los centros y ciclos.

Porque el problema es que ya, si tú quieres Cocina y estás en Parla, tienes que ver a ver dónde está lo más cercano. No es como un instituto, que seguro 
que hay un instituto en Parla, pero a lo mejor en Parla no hay Cocina [...] Lo que planteamos es que haya oferta de todo en todos los sitios. Es decir que de Cocina tengas al menos una oferta mínima en cada una de las direcciones territoriales, sobre todo en Grado Medio, porque uno de Coslada no se va a ir a Getafe a hacer el curso [...] No hay un plan de oferta, vamos. (S-FP)

\subsection{Demanda, ¿de quién? Necesidades del mercado vs. intereses de los estudiantes en FP}

Los representantes de la Administración y la patronal, por su parte, hablan de un «modelo» de FP «joven», en definición, y señalan como principales criterios de planificación de la oferta de FP la demanda por parte de las empresas y, con ello, las posibilidades de inserción laboral de titulados (criterio ausente en Bachillerato), y la demanda del alumnado.

Tenemos dos criterios [...] uno más importante, otro menos importante, que es muy difícil de encaminar, que es: la densidad de titulados de las empresas [...] analizar la inserción laboral de los titulados y conocer las expectativas de puestos que van a ofrecer a las personas para ocuparlos en los próximos años, eso es fundamental. Y también atendemos, segundo, a la demanda de los alumnos. Entonces sí tratamos de organizar una oferta que responda a la inserción laboral real de las empresas, pero siempre muy fijada en la demanda de los alumnos. Es complicado muchas veces porque no cuadran las dos cosas, pero nuestra idea es que los alumnos que salgan de FP tengan unos puestos de trabajo buenos. (CA-FP)

La tensión entre necesidades del mercado e intereses de los estudiantes aparece en los discursos de los entrevistados como un condicionante clave de la planificación de FP. Aunque la relación con el mercado laboral es resaltada por todos los entrevistados como un condicionante esencial de la oferta de FP y un criterio fundamental para su planificación, difieren en el peso que le atribuyen: la Administración y la patronal lo consideran el criterio más importante, por delante de la demanda de los alumnos; los sectores críticos muestran más sensibilidad hacia esta última y critican que se le anteponga la demanda de los sectores económicos. Hay consenso en que no conviene saturar las posibilidades de inserción laboral del alumnado (produciendo un exceso de titulados), pero los actores difieren en el modo de encontrar el equilibrio con los intereses de los jóvenes. Los sectores críticos inciden más en que el alumnado pueda desarrollar sus proyectos profesionales a través de su formación, pero no niegan la importancia del mercado laboral y sus necesidades, que, además, exigirían de la FP una velocidad de actualización que frecuentemente no encaja con los ritmos de las administraciones.

Las FP tienen que ser enseñanzas muy vivas, muy activas [...] tú tienes que ir estudiando el mercado [...] y en función de lo que necesita el mercado ir creando ciclos, enseñanzas, porque, aunque la demanda sea muy alta, tú no puedes ofertar muchas plazas de una determinada especialidad porque estarías 
sacando al mercado titulaciones que luego no podría el mercado absorber. Y el tema burocrático y el tema administrativo son muy lentos, y muchas veces no se va a la misma velocidad que va el mercado [...] muchas veces vamos por detrás de lo que socialmente se necesita. (AD-FP)

Los criterios oficiales (demanda económica y de los estudiantes) pueden chocar, así, con otros más prácticos, como el presupuesto disponible, la existencia de infraestructuras e instalaciones previas, de profesorado o de empresas dispuestas a colaborar, que generan una fuerte inercia institucional.

Hay una estructura de centros y una estructura de formadores con sus cualificaciones y competencias correspondientes, con lo cual eso es una realidad que las consejerías de Educación tienen que manejar. Entonces, a la hora de planificar nuevos grados, ellos de alguna forma tienen que ver si cuentan ya con los profesores con la titulación que se necesite y las competencias o experiencias que se necesiten o si por el contrario tendrían que crear nuevas plazas. Y ahí me imagino que ellos tendrán que jugar con las limitaciones presupuestarias que tengan. También algunos ciclos, en función del tipo, la instalación que se requiere, los equipamientos y demás, pues tampoco son ciclos a lo mejor que se puedan montar de forma inmediata, que requieren un poco a lo mejor de realizar obras en los centros que ya tienen, adquirir la maquinaria para la parte más práctica, o localizar a las empresas que puedan donar esos equipos... (PL)

Esto genera, a su vez, desigualdades entre centros con larga tradición de $\mathrm{FP}$, con más alumnos, docentes y equipamientos, $\mathrm{y}$, por tanto, más recursos para abrir nuevos ciclos y plazas, y otros con menos. Se afirma en algunos casos que los centros privados tendrían menos dificultades para seguir el ritmo del mercado, al contar con más recursos económicos y no depender de la Administración, pero también, según los críticos, esta última aplicaría un doble rasero.

¿Cuántos ciclos autorizas en el ámbito privado-concertado y en el ámbito privado y cuantos autorizas en el ámbito público? Porque lo que sí es verdad es que no puedes cerrar algo público teniendo lo concertado. El objetivo de lo concertado siempre ha sido que, si el ámbito público no llegaba a los sectores de una parte de la sociedad, pues se complementa con el ámbito concertado. Y a veces cierran centros públicos y se mantienen los concertados, eso no puede ser. Entonces el objetivo no es que ahora de repente se abren quince en los públicos, dices: «No, en los públicos no abro más porque saturo el mercado». Ya, pero sí permites que se abran doscientos privados más cinco concertados... [...] No competimos en igualdad de condiciones que los concertados. (AD-FP)

La Administración emplearía el argumento de la saturación del mercado laboral para no autorizar la apertura de nuevos grupos o titulaciones en centros públicos, pero los autorizaría en centros concertados o privados, lo que dejaría a los primeros en desventaja y permitiría a los segundos desarrollar una oferta más diversa o distintiva, con ciclos nuevos y a la última (algunas titulaciones de FP solo se impartirían en centros privados). La tensión entre demanda del 
mercado e intereses de los jóvenes se entrelaza, así, con el conflicto públicaprivada y cómo se concibe el papel de cada una de ellas, y con las desigualdades de recursos entre centros. Esto se traduce, a su vez, en desiguales oportunidades en la elección de los distintos estudios de FP en función del centro en que se esté escolarizado (y su trayectoria, oferta y titularidad), y refuerza el desajuste con las preferencias del alumnado y su movilidad.

\section{La elección de estudios posobligatorios: ¿una decisión libre?}

\subsection{Elegir la vía 'fácil': limitaciones de la oferta y relaciones sociales}

La oferta, con las contradicciones que hemos señalado, condiciona fuertemente las oportunidades y elecciones del alumnado. Cuando la demanda supera la oferta, los entrevistados coinciden en que el alumnado opta por tres vías: otra titulación similar o relacionada, un centro privado, si se lo pueden permitir, o cursar otras enseñanzas. En este tercer caso, la oferta y la demanda de FPGM condicionan la demanda de Bachillerato, de forma que la oferta de ambas enseñanzas no puede entenderse de forma independiente sino interrelacionada, en contraste con su tratamiento institucional diferenciado. En esta situación también influyen las notas de corte en FP: la escasez de plazas y la alta demanda hacen que estas sean muy elevadas en algunos ciclos y que parte del alumnado no pueda acceder a los estudios deseados, de manera que las alternativas son el abandono, la permanencia en Bachillerato, la elección de unos estudios de FP distintos a los deseados inicialmente o la elección de un centro privado.

Todo lo que es Informática tiene una lista de espera y unas notas de corte muy altas, con lo cual los chicos no alcanzan esa nota y acaban en la nada, se quedan perdidos. Pero claro, es que no hay plazas. (AO)

Este filtro académico, paradójico en una enseñanza que no se considera académica y que se asocia en el imaginario colectivo a una menor exigencia que el Bachillerato, tiene efectos perversos: dificulta la transición al Grado Medio, especialmente a quienes tienen dificultades en la ESO, que tendrán menos posibilidades de elegir y de enganche. El Bachillerato puede convertirse entonces en una opción por inercia o por descarte para una parte del alumnado (y no solo a la inversa). Se reitera, así, en las entrevistas el ejemplo de los estudiantes que, ante la falta de otras opciones, continuarían estudiando Bachillerato sin un interés «real» por estos estudios, lo que deriva en un alto fracaso y abandono en el primer curso.

Entonces, esto ha hecho que se resientan un poco también los resultados en esta etapa, porque, verdaderamente, el alumno que sería de un perfil de FP no ha encontrado plaza, y nos encontramos con alumnos que, una vez terminado primero de Bachillerato, tienen que volver otra vez a FP porque les resulta difícil en ese momento. Luego ellos lo pueden sacar posteriormente. (AD) 
Así, la falta de oferta de FP, especialmente de titularidad pública y desigual en el territorio, condiciona también los resultados académicos en posobligatoria e intensifica la discontinuidad de las trayectorias.

Frente a las limitaciones de la oferta, los entrevistados destacan como principal motivo de estos «desajustes» entre la demanda y la oferta, entre los deseos o "perfiles» de los jóvenes y su «elección" posobligatoria, el peso de sus redes de relaciones — su capital social (Bourdieu, 1980) —: los estudiantes primarían el lugar de los estudios (centro o barrio), y con ello su cercanía a sus familias y grupos de pares, y la continuidad con las rutinas, frente al tipo de estudios y su contenido.

El alumnado de Bachillerato, en general, se quiere quedar con sus amigos, en su zona, y eso es muy difícil, es decir, el alumnado no se mueve; si es dentro de la zona y va un grupito de amigos, bien, y hay veces que te encuentras con uno o dos, pero, si no, el alumnado [...] Alumnos que igual piden plaza en un ciclo y no tienen dicen: «¿Y qué tenéis?». «¿Cómo qué tenemos?». «No, es que me quiero matricular». Le da lo mismo, claro, dices, que vayas a hacer Informática y te quedes haciendo Edificación y Obra Civil [...] En Grado Superior ellos seleccionan más y se mueven más, pero a un alumno de Grado Medio le cuesta, y, además, si es por la mañana mejor. (AD-FP)

Las relaciones de amistad y la confianza de las familias en el centro ya conocido generan vinculación con el barrio y la institución, especialmente a los 16-17 años. Este apego es minusvalorado por los agentes políticos, que conciben la elección de estudios en términos estrictamente racionales, y refuerza en una parte del alumnado una estrategia de no movilidad y una "elección» de estudios «desajustada» a los deseos, las capacidades y las posibilidades que se les atribuyen, o a sus propias preferencias académicas o profesionales, en tanto que lo contrario implicaría movilidad geográfica, un cambio de entorno escolar y social, en las relaciones sociales y en las rutinas cotidianas, que no necesariamente estarían dispuestos a afrontar.

\section{2. "Primero va la universitaria»: la persistente diferencia de prestigio entre Bachillerato y FP}

Otro de los condicionantes fundamentales de la elección de rama de posobligatoria, según los entrevistados, es la persistencia de prejuicios sociales sobre la FP. La elevación progresiva de las expectativas escolares de la población es interpretada por varios entrevistados como «titulitis» y se deslegitima que la mayoría de los padres quieran para sus hijos un título universitario como una patología, un deseo irracional que no se correspondería con las capacidades y posibilidades reales de los alumnos, especialmente de aquellos con más dificultades.

Sigue estando mal visto decir: «Mi hijo va a FP». En general. Es que, claro, si tu hijo va al Bachillerato y tu hijo va a la universidad, es como de más caché. 
En mi caso, mi hijo ha hecho FP. Entonces, claro, eso siempre queda como muy llamativo cuando los padres dicen: «Es que yo quiero no sé qué». Perdona, yo, como tú, quiero lo mejor para mi hijo. Mi hijo no quería estudiar, mi hijo quería no sé qué. Y entonces, aquí hay dos opciones: o te impones a un tipo de 16 años y dices «estudia porque lo digo yo», o bien negocia con tu hijo, es adolescente, tiene 16, mira qué intereses tiene y poco a poco va dando su vuelta, su giro, su no sé qué, y la vida le sitúa donde le tiene que situar. En la universidad, en la FP o en lo que sea. (AO)

La peor imagen de la FP entraría en contradicción con las posibilidades reales que ofrece al alumnado que tiene más dificultades o madura más tardíamente (que podría acceder a la universidad más adelante), o con las mayores opciones de inserción laboral que abre (frente a la sobrecualificación de los universitarios, que ocuparían puestos de titulados de Grado Superior o cursarían ciclos superiores para acceder a una mayor inserción laboral). Este distinto prestigio no sería, por tanto, cuestión únicamente de percepción e imagen - que podría corregirse con información o campañas de comunicación, según la interpretación dominante_-, sino también de la realidad de los puestos de trabajo y salarios a los que permite acceder cada vía (Merino et al., 2011).

O sea, todos los orientadores, como todos los profesionales que estamos en los departamentos de orientación o mayoritariamente, y el caso de los profes que abrumadoramente piensan que "si vales para estudiar, al Bachillerato", es decir, si tú eres capaz de retener contenidos, si tienes capacidad de memorizar y tal, Bachillerato [...] Es decir, no piensas en términos de profesión, piensas en términos de reconocimiento social, que, a su vez, [...] es reconocimiento laboral y económico [...] Ese que hace Bachillerato y luego hace una carrera y a lo mejor le importa una mierda, al final es más contratado para determinados puestos que los de FP. Pues es que, claro, se produce como un trasvase, o sea, vuelven a la FP por la vía laboral. (S-FP)

Además, la diferencia de prestigio se mantendría por las prácticas de orientación formal e informal (sobre todo) que se dan desde los propios centros educativos en ESO, donde los profesionales (docentes, orientadores) tenderían a reproducir la hegemonía del Bachillerato. En este sentido, los esquemas y las concepciones de los profesionales, la "cultura de centro» o su habitus institucional condicionan fuertemente las elecciones del alumnado (Tarabini et al., 2018).

La FP, desde hace mucho tiempo, ha sido el cajón de sastre: el alumno que no sirve para la universidad, parece que de alguna forma peyorativa, pues hacía la FP. Todavía hay muchos centros de secundaria en Madrid en donde esa postura la tienen las direcciones. Aquí el que vale es el que va a la universidad, el otro es que es un alumno mediocre [...] Siguen todavía acogidos a esa imagen, es decir, «nosotros preparamos para la facultad», y a veces se lo dicen a los padres. Y cuando les dices: "Pero bueno, dale opción de FP, a veces el alumnado no se atañe a esa dinámica de trabajo que tenéis en los centros, y eso no significa 
que el alumno sea... sino que a lo mejor necesitaría otras propuestas de trabajo, otras dinámicas en los centros para poder alcanzar los mismos objetivos, cuando estéis dispuestos a cambiarlas». Entonces, bueno, siguen utilizando esa forma. Primero va la universitaria. (AP)

Esta posición de algunos equipos de secundaria se vería reforzada, según los entrevistados, por su desconocimiento de las enseñanzas de FP, y sería menos común en centros que tienen también oferta de FP.

No obstante, los entrevistados señalan que, aunque la imagen tradicional de la FP persiste, parece estar cambiando: frente a los tiempos de la Ley General de Educación, en los que todos concuerdan en situar la peor valoración de la FP (concebida como vía para quienes no obtenían el título de EGB), ${ }^{4}$ existiría en la actualidad una mejor concepción de la formación y las posibilidades laborales que ofrece.

Creo que entre el alumnado está cambiando, y están eligiendo bastante FP. Porque el tema de la formación yo considero que es de bastante calidad, y lo digo por experiencia [...] Parte del alumnado sí que está viendo que la facultad ahora, es cierto, es un nivel académico y cultural importante, pero que no te ofrece las garantías luego hacia el mundo laboral como a lo mejor en la FP, alguna sí, con esa formación más específica. Entonces, sí que hay mucho alumnado ya que acaba y que a lo mejor hace Bachillerato y que inicia en la facultad y se dirige luego a la FP. (AF)

También por parte de las familias se observa un cambio en la valoración de la FP, si bien sigue habiendo muchas resistencias que se achacan fundamentalmente, además de a la presión social, a la falta de conocimiento sobre estas enseñanzas.

En el instituto anterior, tenía cinco familias profesionales y una de las cosas que hacíamos era pues la típica visita guiada por grupito y pasaban por los distintos talleres, y cuando un padre llegaba y veía que para prótesis había un material de laboratorio y veía los sillones de dentista y veía todo el equipamiento, decía: «AAh, esto está en el instituto!». Pues claro, esto está en el instituto, lo mismo que está el taller de madera con una máquina para cortar tableros, no sé qué. «Ah, y eso también!». Es como que la FP sigue siendo una gran desconocida. Creo que depende de la información que hayan recibido los padres. Cuando tú haces ese tipo de vuelta y van viendo lo que hay, los padres están más abiertos a FP. Yo creo que hay una parte que, por mucho que nos lo metan en la tele, si el papá o la mamá no van al sitio y ven lo que hay, el cacharreo que tienes y lo que significa... (AO)

Este aumento en la valoración de la FP también se aprecia en la Administración educativa, si bien de forma sutil y más de palabra que de acción. No obstante, esta mejora de imagen no se habría traducido, por el momen-

4. Educación General Básica, antigua etapa obligatoria que abarcaba de los 6 a los 14 años. 
to, en mejores resultados académicos, con un alto abandono escolar entre el alumnado de FPGM, aspecto que puede estar relacionado con su trayectoria anterior, dado que las calificaciones obtenidas en etapas anteriores son uno de los principales predictores de los resultados en la posobligatoria (Bernardi y Cebolla, 2014).

Las titulaciones que tenemos no son muy bajas, y normalmente no se nos pierden muchos, pero siempre hay un $20 \%$ de alumnos por ahí que pierdes. De este $80 \%$ y tal, hay algunas que sacan el Bachillerato por el nocturno, así que podemos hablar de un $85 \%$ de alumnos que terminan el Bachillerato. Y de los que se desvían, muchos se reenganchan otra vez; aquí es donde entra la escuela de adultos. Los pintas te vienen a pedir información para finalizar los estudios porque se han dado cuenta de que, si acaban, en su puesto de trabajo ganarían más dinero. El nivel socioeconómico es un nivel bajo, y muchas veces la información que puedan tener los padres no es la que pueden tener los padres que tienen otro nivel diferente. $(\mathrm{AD})^{5}$

Si bien buena parte de los entrevistados afirman desconocer las causas de este abandono en FP, apuntan como un posible factor la falta de oferta, motivo por el cual el alumnado se matricula en titulaciones que considera afines a las que inicialmente quería cursar, pero que después se revelan como diferentes de lo que imaginaba. Pero insisten especialmente en la orientación, que aparece a la vez como problema y solución: las familias y alumnos elegirían «mal», al desconocer sus opciones y guiados por prejuicios; los deseos de los alumnos serían volubles e influenciables, condicionados fuertemente por los amigos, las modas y los medios de comunicación; la solución sería reconducirles a través de una "correcta» orientación. No obstante, algunos señalan otros factores, como la falta de ajuste entre el nivel de conocimientos previo del alumnado y el nivel de exigencia de las enseñanzas, la «inmadurez» del alumnado o la falta de criterios ajustados a la hora elegir estudios, donde primarían información procedente de experiencias y fuentes informales (familiares, amigos, etcétera).

El sistema educativo está fracasando con los grados medios. Por lo menos, yo lo que sé en Madrid y alguna otra zona, es que la mitad del alumnado que empieza fracasa. ¿Razones? Básicamente, que no eligen lo que quieren, eligen lo que pueden [...] El típico ejemplo sería: «Mi prima ha hecho Peluquería». [...] ¿Luego le gusta o no le gusta la peluquería? ¿Sabe lo que es el trabajo en la peluquería? Estar allí las horas que tienes que estar... Cuando descubren lo que es eso de verdad, pues lo dejan [...] Luego, por otro lado, claro, eso se resolvería si hubiese suficiente oferta y mucha mejor orientación, que no la tienen. Para Grado Medio, es horrible, porque no está bien conceptuado. En

5. Aunque no hay disponibles datos oficiales sobre abandono escolar en Bachillerato y FP para la $\mathrm{CM}$, sí sabemos que, en Bachillerato, el 15,3 \% no promociona en primer curso (13,9\% en la capital) y el 20,1 \% no titula en segundo curso (17,9\% en la capital). En FPGM, solo se ofrecen los datos de alumnos de segundo curso que no titulan: el 34,3\% (31,3\% en la capital) (Comunidad de Madrid, 2018: 464-468), pero esto no refleja el abandono en primero. 
Bachillerato, un poquito para Grado Superior, porque ya tienes un grado de madurez mayor, pero es que el problema es que para ir al Grado Medio te vas con 16 años, con 16 o 15 años no tienen madurez para decidir. A veces deciden los padres y deciden por criterios de grupo primario, es decir, porque ha ido mi primo, porque mi padre me ha dicho que esto va bien, porque ha leído en el periódico que esto va a funcionar, pero la vocación no se suele tener en cuenta, y eso es fundamental porque no estás 2.000 horas de tu vida en algo que no te guste. $\mathrm{O}$ sea, al cabo del primer año, lo dejas. Y luego, el otro fracaso es porque el nivel académico que se pide es demasiado bajo para lo que ofrecen ahora mismo a los alumnos. Y esta solución no es nada sencilla. La fácil sería decir "pues bajemos en nivel», pero claro tú tienes que ofrecer un nivel 2 y tienes que ofrecer una mínima formación generalista en términos de física [...] (S-FP)

La FP, especialmente en Grado Medio, se encuentra atravesada por la tensión entre dos concepciones distintas de sus funciones: una social (recuperar a los alumnos con dificultades) y otra profesionalizadora (formar profesionales cualificados para el mercado de trabajo) (Merino, 2013; Tarabini y Jacovkis, 2019). La primera, dominante en el nivel más bajo y menos prestigioso de FP (FP Básica), contrasta con el predominio de la segunda en FP Superior, su nivel más valorado y prestigioso, lo que genera una fuerte ambivalencia en su nivel medio (reforzada por su conexión con FP Básica, que es vista por algunos entrevistados como una fuente de devaluación del Grado Medio al facilitar el acceso de alumnos con «bajo nivel»). Además de esta diferenciación vertical en FP, encontramos otra horizontal, entre familias y ciclos de distinto prestigio, que genera desajustes entre su nivel de demanda y su nivel inserción laboral: se reitera en las entrevistas la idea de que los jóvenes que acceden a FPGM (a pesar de provenir en gran medida de clases populares) tienden a evitar los ciclos asociados con profesiones obreras tradicionales (el trabajo de "mono azul»), a pesar de su alta inserción laboral (sería el caso de Electricidad o Soldadura), y a demandar familias y ciclos con mucha demanda y baja inserción laboral (se citan habitualmente Cocina, Video Disc-Jockey, Actividades Deportivas, Informática, con más solicitudes que plazas en Madrid) (CCOO Madrid, 2019: 44-45).

\subsection{Barreras y sesgo de clase}

Frente a otros factores, los económicos (precio de las enseñanzas y costes asociados) no aparecen en los discursos de los entrevistados como un factor decisivo de las elecciones, salvo en el caso de la FP Superior, donde las tasas son elevadas. No obstante, aparecen algunas discrepancias: mientras unos afirman que no se trata de un factor determinante para la elección entre Bachillerato y FPGM, ya que ambas enseñanzas son gratuitas en centros sostenidos con fondos públicos, otros destacan que el coste de los materiales, según la familia de FP, puede ser superior en las enseñanzas de Bachillerato.

Hay especialidades, por ejemplo Peluquería, que les hacen comprar su secador y sus peines o, en Cocina, les hacen comprar su estuche de cuchillos o lo que 
sea, pero, en general, en los centros públicos el coste que tienen es 1,12 €, luego de libros de texto generalmente no hay muchos y el coste es bajísimo [...] Bachillerato es más caro, los libros de texto son caros [...] Te pueden salir por unos 300 o 400 euros. (AD)

Además, las ayudas económicas para realizar estudios de FP de Grado Medio son escasas en la CM: solo existe la beca general del Ministerio de Educación, no existe ninguna beca autonómica (salvo la beca-cheque para escolarización en Grado Superior en centros privados). Pese a las discrepancias sobre la influencia directa de los factores económicos, algunos entrevistados — pocos, críticos y docentes de FP_ constatan el peso determinante de factores socioeconómicos y culturales y el sesgo de clase en la separación de la rama académica y la rama profesional, que tienden a permanecer ocultos bajo las diferencias académicas.

En Grado Medio, yo creo que sí que se nota, porque es un puesto de ayudante, entonces socialmente es una baja cualificación [...] es semicualificado, pero para los efectos de la interpretación de las familias eso es de baja cualificación. Entonces, solo va gente que mentalmente o en su proyección es de clase trabajadora, con menos aspiraciones. Si tienes más aspiraciones, lógicamente tienes que aspirar al Bachillerato. Sí, eso está claro. Ahí va a ser difícil que la FP deje de ser para trabajadores. O sea, el hijo de un profesor o el hijo de un ingeniero es difícil que acabe en [...] Haría FP de Grado Superior y unas muy específicas, todo lo demás no. Si haces Animación 3D sí, porque, claro, simbólicamente es hacer juegos, y te forras además porque esa industria mueve mucho dinero. (S-FP)

Este sesgo de clase se manifestaría de forma distinta según los niveles de FP, y es especialmente intenso en FP Básica y FPGM, pero menos evidente en FP Superior por su mayor valoración social y su alumnado más maduro y socialmente diverso (con menor composición obrera y más presencia de clase media), especialmente en ciclos más prestigiosos y distintivos.

En Grado Superior, hay mucha gente de nivel socioeconómico medio, incluso alto, que, bueno, decide, arranca en la universidad y decide volver porque no era lo suyo, tiene el Bachillerato, pero en Grado Medio sigue el perfil, vamos, y más ahora que vienen de la FP Básica [...] El ámbito social sigue siendo bajo todavía, más en Grado Medio que en Grado Superior, eh. Hombre tampoco es extremo, también depende mucho del barrio donde estés, porque en Grado Medio sí recoges bastante de la zona, y en Grado Superior no, en Grado Superior seguramente que el $80 \%$ - $90 \%$ no son del barrio, sobre todo en el ámbito de $\mathrm{FP}$, que somos cuatro en todo Madrid, pues te vienen de todos los extrarradios. (AD-FP)

Las divisiones de clase parecen, por tanto, guardar relación con las diferencias de prestigio entre las distintas ramas de posobligatoria y en el interior de la rama profesional (entre distintos niveles, familias y ciclos), y no tienen que ver única- 
mente con factores económicos, sino también con factores culturales y simbólicos (creencias, expectativas y aspiraciones, imagen y prestigio de ciertas enseñanzas y ocupaciones) e institucionales (diseño de la oferta y el proceso de admisión, prácticas de orientación formal e informal en ESO) que se entremezclan.

\section{Conclusiones}

La transición a la educación posobligatoria, momento crucial en las trayectorias escolares de los jóvenes, no es independiente de los distintos factores políticos e institucionales que operan en cada contexto local. En un entono como el madrileño, caracterizado por una alta concentración de población y de centros escolares, con una alta proporción relativa de población joven que alcanza la educación posobligatoria, un gran predominio de la rama académica sobre la profesional y un fuerte peso de la oferta privada, la ideología de la «libertad de elección" choca con la realidad de una etapa educativa posobligatoria en la que las posibilidades de acceso y las «elecciones» de los jóvenes están fuertemente condicionadas por distintos factores. Como muestran los resultados de este estudio, a pesar de su fuerte diferenciación institucional, material y simbólica, las enseñanzas de Bachillerato y FP no pueden entenderse de forma independiente: si su diseño institucional sigue pautas y criterios diferenciados (en su administración y gestión, en los requisitos de acceso, en la organización del proceso de admisión), ambas se encuentran estrechamente relacionadas, directa e indirectamente, en las elecciones de los jóvenes.

Además, entre los condicionantes del acceso a la educación posobligatoria, los factores políticos e institucionales se revelan como un elemento clave. La falta de oferta, especialmente de titularidad pública, así como la desigual distribución territorial de las enseñanzas, en el caso de la FPGM, y del Bachillerato para la modalidad de Artes, ofertada en pocos centros, condicionan fuertemente las oportunidades de acceso de los estudiantes, lo que implica para ellos una movilidad obligada que no necesariamente están dispuestos a llevar a cabo. Esto generaría efectos paradójicos con consecuencias perversas, como que estos alumnos, debido a la insuficiente oferta, acaben engrosando el Bachillerato en sus centros de origen o en centros cercanos a su domicilio a pesar de su deseo inicial de matricularse en un ciclo formativo, o que opten por un ciclo distinto al deseado, con las consiguientes posibilidades de desajuste, fracaso y abandono. La falta de oferta pública también generaría un trasvase de matrícula a los centros privados, lo que implicaría una brecha en la posibilidad de acceso a una formación ajustada a los intereses profesionales y personales de los estudiantes entre los que pueden permitirse el pago de los costes de un centro privado y los que no.

Pese a la relación entre ambas enseñanzas, se aprecian también características específicas. En el caso de Bachillerato, se da una doble naturalización: por un lado, se presenta como la continuidad «natural», como la opción "fácil» (Tarabini y Jacovkis, 2019: 249), al depender de una misma dirección general que la ESO, de un mismo perfil del profesorado y en muchos casos ofertarse en el mismo centro; por otro, el diseño de estas enseñanzas, de corte fuertemente 
académico, permanece ajeno a análisis y debate, así como libre de las presiones del mercado, que pesan fuertemente en la rama profesional. La planificación de la oferta se realiza centro a centro y en función de la demanda, lo que se traduce en una optatividad limitada y en desiguales posibilidades de diversificación de la oferta entre centros grandes y pequeños.

La FP, por el contrario, aparece como la opción «difícil» (ibid.), dependiente de otra dirección general (teóricamente para ponerla en valor), que requiere en muchos casos un cambio de centro respecto a la ESO (por la ausencia de los ciclos formativos deseados o, directamente, de oferta de FP en el centro de origen), un cambio en la cultura y las rutinas escolares, y en el prestigio de la enseñanza cursada y de los oficios a los que da acceso. Existe consenso entre los actores políticos, educativos y empresariales en que su planificación debe regirse por dos criterios principales: la demanda de las empresas y la inserción laboral de titulados (ausente en Bachillerato), y la demanda del alumnado. No obstante, ambos criterios entran con frecuencia en tensión y existen disensos sobre el peso que debe tener cada uno de ellos, aunque los policymakers tiendan a priorizar el primero. Además, en la práctica, el diseño de la oferta de FP se caracteriza por la ausencia de un plan a largo plazo, lo que se traduce en actuaciones desagregadas a nivel de centro, sin un criterio territorial o de conjunto, y en muchas ocasiones condicionadas por la existencia previa de recursos (instalaciones, equipamientos y personal). El criterio de ahorro económico tiende a producir concentración de la oferta, y genera mayores desigualdades en un diseño institucional y territorial de por sí desigual debido a la diversidad de familias existentes y a las diferencias de coste económico entre ellas.

Las entrevistas apuntan, además, a una persistencia de la valoración negativa de la FP frente al Bachillerato, especialmente en el caso del Grado Medio, aún asociada a un alumnado menos "hábil» y "académico», con dificultades, y vinculada también a profesiones de menor cualificación y prestigio, a pesar de su mayor inserción laboral. Habría cambiado, a pesar de todo, su imagen desde los años 80 en el sentido de una mejora. Los agentes perciben la intención de dotarla de mejor posición en el sistema educativo, pero siguen definiéndola como la gran olvidada y una gran desconocida para las familias, el alumnado y los propios profesionales de la educación (directores, profesorado, orientadores, etcétera). Pese a este ligero cambio, se mantiene la concepción de la FP como una opción «de baja calidad para estudiantes de baja calidad» (Calero, 2008: 58), marcada por la tensión entre su función social y su función profesionalizadora, aunque con diferencias importantes de prestigio tanto desde una perspectiva vertical, entre sus niveles Básico, Medio y Superior (con fuerte ambivalencia del GM al situarse en medio), como desde una perspectiva horizontal, en función de las familias y ciclos. La comprensión de las desigualdades que se producen tras la educación obligatoria requiere tener en cuenta el peso de los factores políticos e institucionales, y en concreto de la configuración de la oferta y el diseño institucional de las enseñanzas, que participan, en interacción con factores sociales, subjetivos, territoriales y culturales, en la construcción de las elecciones y transiciones educativas. 


\section{Financiación}

Este artículo forma parte del proyecto La construcción de las oportunidades educativas post-16. Un análisis de las transiciones a la educación secundaria posobligatoria en contextos urbanos (EDUPOST16, Ref. CSO2016-80004-P), financiado por el Ministerio de Economía y Competitividad, que se desarrolla en paralelo en Barcelona y Madrid. Una versión previa se presentó en la XX Conferencia de Sociología de la Educación de 2018.

\section{Referencias bibliográficas}

BALL, Stephen J. (2006). Education policy and social class: The selected works of Stephen Ball. Londres: Routledge.

Ball, Stephen J.; Maguire, Meg; Macrae, Sheila (2000). Choice, Pathways and Transitions Post-16. New youth, new economies in the global city. Londres: Routledge. $<$ https://doi.org/10.4324/9781315043296>.

Ball, Stephen J.; Maguire, Meg; Braun, Annette (2012). How schools do policy. Policy enactments in secondary schools. Londres: Routledge. $<$ https://doi.org/10.4324/9780203153185>.

Bernardi, Fabrizio; Requena, Miguel (2010). «Inequality in educational transitions: the case of post-compulsory education in Spain». Revista de Educación, número extraordinario, 1, 93-118.

Bernardi, Fabrizio; Cebolla, Héctor (2014). «Clase social de origen y rendimiento escolar como predictores de las trayectorias educativas». Revista Española de Investigaciones Sociológicas, 146, 3-22. $<$ https://doi.org/10.5477/cis/reis.146.3>.

Bonal, Xavier (2012). «Education policy and school segregation of migrant students in Catalonia: the politics of non-decision-making». Journal of Education Policy, 27 (3), 401-421. <https://doi.org/10.1080/02680939.2011.645168>.

Bourdieu, Pierre (1980). «Le capital social. Notes provisoires». Actes de la Recherche en Sciences Sociales, 31, 2-3.

Brunila, Kristiina; Kurki, Tuuli; Lahelma, Elina; Lehtonen, Jukka; Mietola, Reetta; Palmu, Tarja (2011). «Multiple Transitions: Educational Policies and Young People's Post-Compulsory Choices». Scandinavian Journal of Educational Research, 55 (3), 307-324. <https://doi.org/10.1080/00313831.2011.576880>.

Calero, Jorge (2008). «Problemas en el acceso a la educación postobligatoria en España». Revista de la Asociación de Sociología de la Educación (RASE), 1 (1).

Calero, Jorge; Choi, Álvaro; Waisgrais, Sebastián (2010). «Determinantes del riesgo de fracaso escolar en España: una aproximación a través de un análisis logístico multinivel aplicado a PISA-2006». Revista de Educación, número extraordinario $1,225-256$.

CCOO Madrid (2019). Diagnóstico de la educación en la Comunidad de Madrid. Madrid: CCOO-Madrid.

Comunidad de Madrid (2018). Datos y cifras de la educación 2018-2019. Madrid: Consejería de Educación e Investigación.

- (2019). Informe 2018 sobre el sistema educativo en la Comunidad de Madrid. Curso 2016-2017. Madrid: Consejo Escolar de la CM. 
Decreto 29/2013, de 11 de abril, del Consejo de Gobierno, de libertad de elección de centro escolar en la Comunidad de Madrid (BOCM de 12 de abril de 2013).

— 72/2015, de 7 de julio, del Consejo de Gobierno, por el que se modifica la estructura orgánica de las consejerías de la Comunidad de Madrid (BOCM de 8 de julio de 2015).

- 11/2019, de 5 de marzo, del Consejo de Gobierno, por el que se modifica el Decreto 29/2013, de 11 de abril, de libertad de elección de centro escolar en la Comunidad de Madrid (BOCM de 11 de marzo de 2019).

Dupriez, Vincent; Dumay, Xavier (2006). «Inequalities in school systems: effect of school structure or of society structure?». Comparative Education, 42 (2), 243-260. <https://doi.org/10.1080/03050060600628074>.

Elias, M.; Daza, L. (2017). "¿Cómo deciden los jóvenes la transición a la educación postobligatoria? Diferencias entre centros públicos y privados-concertados». Revista de la Asociación de Sociología de la Educación, 10 (1), 5-22.

Lahelma, Elina (2009). «Dichotomized Metaphors and Young People's Educational Routes». European Educational Research Journal, 8 (4), 497-507. <https://doi.org/10.2304/eerj.2009.8.4.497>.

Martín Criado, Enrique (2010). La escuela sin funciones. Barcelona: Bellaterra.

Merino, Rafael (2013). «Las sucesivas reformas de la formación profesional en España o la paradoja entre integración y segregación escolar». Archivos Analíticos de Políticas Educativas, 21 (1). <https://doi.org/10.14507/epaa.v21n66.2013>.

Merino, Rafael; García, Maribel; Casal, Joaquim; Sánchez, Albert (2011). «Itinerarios formativos y laborales de los jóvenes graduados en Formación Profesional. Sobre algunos prejuicios en la formación profesional». Sociología del Trabajo, 72, $137-155$.

Ministerio de Educación y Formación Profesional (2019a). Las cifras de la educación en España. Estadísticas e indicadores. Edición 2019. Madrid: MEFP.

- (2019b). Datos y cifras. Curso escolar 2019/2020. Madrid: MEFP.

Murillo, F. Javier; Martínez-Garrido, Cynthia (2018). «Magnitud de la segregación escolar por nivel socioeconómico en España y sus Comunidades Autónomas y comparación con los países de la Unión Europea». RASE. Revista de Sociología de la Educación, 11 (1), 37-58.

<https://doi.org/10.7203/RASE.11.1.10129>

Orden 2509/2013, de 1 de agosto, de la Consejería de Educación, Juventud y Deporte, por la que se regula el procedimiento de admisión de alumnos en centros sostenidos con fondos públicos para cursar Formación Profesional de Grado Medio (BOCM de 9 de agosto de 2013).

Prieto, Miriam; Villamor, Patricia (2012). "Libertad de elección, competencia y cali-dad: las políticas educativas de la Comunidad de Madrid». Profesorado. Revista de Curriculum y Formación de Profesorado, 16 (3), 149-166.

- (2018). «El impacto de una reforma: Limitación de la autonomía, estrechamiento de la libertad y erosión de la participación». Archivos Analíticos de Políticas Educativas, 26 (63). $<$ https://doi.org/10.14507/epaa.26.3255>.

Ruiz, María Auxiliadora; Sancho, Miguel Ángel; Esteban, Mercedes de (2019). Indicadores comentados sobre el estado del sistema educativo español 2019. Madrid: Fundación Ramón Areces y Fundación Europea Sociedad y Educación. 
Shavit, Yossi; Müller, Walter (2000). «Vocational secondary education». European Societies, 2 (1), 29-50. <https://doi.org/10.1080/146166900360710>.

Tarabini, Aina; Montes, Alejandro (2015). «La agenda política contra el abandono escolar prematuro en España: la LOMCE contra las evidencias internacionales». Avances en Supervisión Educativa, 23.

Tarabini, Aina; Ingram, Nicola (eds.) (2018). Educational Choices, Transitions and Aspirations in Europe: Systemic, Institutional and Subjective Challenges. Abingdon: Routledge. <https://doi.org/10.4324/9781315102368>.

Tarabini, Aina; Curran, Marta; Castejón, Alba; Montes, Alejandro (2018). «Framing youth educational choices at the end of compulsory schooling: the Catalan case». En: Tarabini, Aina; Ingram, Nicola (eds.). Educational Choices, Transitions and Aspirations in Europe: Systemic, Institutional and Subjective Challenges, 95-109. Abingdon: Routledge. <https://doi.org/10.4324/9781315102368-6>.

Tarabini, Aina; Jacovkis, Judith (2019). «Transicions a l'educació secundària postobligatòria a Catalunya». En: Riera, Jordi (dir.). Reptes de l'educació a Catalunya. Anuari 2018, 235-290. Barcelona: Fundació Jaume Bofill.

van Zanten, Agnès; Olivier, Alice; Oller, Anne-Claudine; Ulhy, Katrina (2018). "National framing and local reframing of students' transition to higher education in France: limitations and pitfalls». En: TARABini, Aina; Ingram, Nicola (eds.). Educational Choices, Transitions and Aspirations in Europe: Systemic, Institutional and Subjective Challenges, 149-166. Abingdon: Routledge. <https://doi.org/10.4324/9781315102368-9>.

Walther, Andreas (2006). "Regimes of youth transitions. Choice, flexibility and security in young people's experiences across different European contexts». Young. Nordic Journal of Youth Research, 14 (2), 119-139. <https://doi.org/10.1177/1103308806062737>. 